\title{
Innovative Treatment for Complex Fractures of Calcaneum by Minimally Invasive Surgery
}

\section{Aditya K. Agrawal', Manish R. Shah ${ }^{1}$, Krunal Shah'2, Malkesh Shah', Sarvang Desai ${ }^{4}$, Jagdish J Patwa ${ }^{4}$}

\begin{abstract}
'Associate Professor, Department of Orthopaedics, Dhiraj Hospital, Smt. BK Shah Medical Institute \& Research Centre, Sumandeep Vidyapeeth Deemed to be University, Piparia Vadodara, Gujarat 391760 India; 'Senior Resident, Department of Orthopaedics, Dhiraj Hospital, Smt. BK Shah Medical Institute \& Research Centre, Sumandeep Vidyapeeth Deemed to be University, Piparia Vadodara, Gujarat 391760 India; ${ }^{3}$ Assistant Professor, Department of Orthopaedics, Dhiraj Hospital, Smt. BK Shah Medical lnstitute \& Research Centre, Sumandeep Vidyapeeth, Deemed to be University, Piparia Vadodara, Gujarat 391760 India; 4Professor, Department of Orthopaedics, Dhiraj Hospital, Smt. BK Shah Medical Institute \& Research Centre, Sumandeep Vidyapeeth Deemed to be University, Piparia Vadodara, Gujarat 391760 India.
\end{abstract}

\section{ABSTRACT}

Introduction: Complex fractures of calcaneum are one of the most difficult fractures to treat for an orthopaedic surgeon. Persistent pain, oedema and broadening of the heel are common complications postoperatively. The treatment is controversial and none of it is ideal. Results of fixation with the calcaneal plate are not satisfactory and other surgical treatments can't prevent subtalar collapse.

Methods: Comminuted, complex, depressed fracture of the calcaneum is elevated with Steinman pin insertion which is used as a joystick. If necessary, the cortico-cancellous screw is pushed to support the subtalar joint. The vacuum is created by elevation within the shell of the calcaneum which is pierced by the guidewire and then the DHS reamer is passed from posterior to the anterior portion of the calcaneum to shift the cancellous portion of the bone in the desired area. The harvested fibular stud graft is pushed from posterior to the anterior aspect of calcaneum over a guidewire to support the gap. So the fibula acts as a supporting stud as well as the cortical cancellous graft.

Results: The criteria for patient-related outcome measures include a reduction in pain, correction of the broadened heel, tiptoes and heel touch standing, painless walking and radiological and clinical healing of the fracture, correction of subtalar depression. Out of twenty-seven patients, we have achieved excellent results in twenty-six patients and good result in one patient.

Conclusion: This is our innovative minimally invasive technique for tackling comminuted complex depressed fractures of the calcaneum. Fibular stud graft helps in packing the gap, providing stud support as well as cancellous grafting which is giving excellent results of maintaining the heel contour of a proper size, relieving the pain early and providing a virtually good range of movement.

Key Words: Calcaneum, Fibula graft, Minimally invasive

\section{INTRODUCTION}

Complex fractures of calcaneum are one of the most difficult fractures to treat for an orthopaedic surgeon. Persistent pain, oedema and broadening of the heel are common complications post operatively ${ }^{1}$. The treatment is controversial and none of it is ideal. Results of fixation with the calcaneal plate are not satisfactory and other surgical treatments can't prevent subtalar collapse.

Calcaneum fracture is disabling and painful injury as it involves heel, which is a weight-bearing portion of the body.
Such injuries are usually due to falling from a height. It causes widening, shortening and deformation of the heel. Fractures of calcaneum account for 1-2\% of all the fractures and are the most common of the tarsal bones fracture. The injury is predominantly in young or middle-aged men. Intraarticular fractures account for approximately $75 \%$ of calcaneum fracture. These are commonly found with injuries involving tibial plateau, pelvis and lumbar spine. So, such injuries involve long term morbidity, long periods of loss of work and income. So ultimately involves socio-economic issues. Though the injuries are common, final treatment has

\section{Corresponding Author:}

Dr. Manish R. Shah, Associate Professor, Department of Orthopaedics, Dhiraj Hospital, Smt. BK Shah Medical Institute \& Research Cen tre, Sumandeep Vidyapeeth, Piparia Vadodara, Gujarat 391760 India; Mob: +91 9825452010; Email: manishshah2001in@yahoo.co.in

ISSN: 2231-2196 (Print)

Received: 22.07 .2020
ISSN: 0975-5241 (Online)

Revised: 18.08 .2020
Accepted: 20.10 .2020
Published: 12.11 .2020 
too many options and confusions. Better imaging techniques have helped a lot in better methods of fixation. Good postoperative rehabilitation has helped in achieving remarkable improvement in the result.

Thus, we need a study to compare the outcome of management of intra-articular calcaneum fractures and to decrease the complication rates of these devastating injuries. We aim to analyse overall results of operated cases of fracture calcaneum, to compare with other series regarding the operative treatment, to compare the result to various surgical methods and to study complications associated with intra-articular fracture calcaneum.

\section{MATERIALS AND METHODS}

The study was carried out at tertiary care hospital from January 2015 till December 2017. All the patients were operated by a single senior orthopaedic surgeon. The study was done after proper consent from the patient and approval from the Institutional Ethics Committee. The patients were operated supine on the simple radiolucent orthopaedic table under image intensifier as shown in Figure 1a and Figure 1b. All patients were given spinal anaesthesia and were operated under tourniquet. One $\mathrm{cm}$ skin incision was made over the tip of the heel under image intensifier. Another skin incision was made on the lateral aspect of the affected leg above the syndesmosis to harvest the non- vascularized fibula bone graft. The comminuted, complex, depressed fracture of calcaneum was elevated with Steinman pin insertion which was used as a joystick. If necessary, cortical- cancellous screw was pushed to support the subtalar joint. Vaccum was created by elevation within the shell of the calcaneum which was pierced by the guidewire. A Steinmann pin was inserted in the anterior part of the calcaneum. Thereafter DHS reamer was passed from posterior to the anterior portion of the calcaneum to shift the cancellous portion of the bone in the desired area as shown in Figure 2a. The harvested fibular stud graft was pushed from posterior to the anterior aspect of calcaneum over a guidewire to support the gap. So the fibula acts as a supporting cortical cancellous bone graft scaffold as shown in Figure $2 b$.

\section{RESULTS}

Total twenty-seven patients were operated. Preoperative lateral and axial views as shown in Figure 1a \& 1 b were taken in all patients. The criteria for patient-related outcome measures include a reduction in pain, correction of the broadened heel, tiptoes and heel touch standing, painless walking and radiological and clinical healing of the fracture, correction of subtalar depression as shown in Figure $3 a$ and Figure $3 b$. Out of twenty-seven patients, we have achieved excellent results in twenty-six patients and good result in one patient as shown in Figures 4 and 5. We also achieved satisfactory radiological outcome as shown in Table 1. One patient with good result had superficial wound infection which subsided subsequently with the short course of intravenous antibiotics given for one week followed by oral antibiotics for two weeks.

\section{DISCUSSION}

This is our innovative minimally invasive technique for tackling comminuted complex depressed fractures of the calcaneum. By elevation of subtalar joint as well as packing of the vacuum created by the elevation of the subtalar joint to support rigidly with fibula graft within the shell of the calcaneum and displacing the cancellous tissue within the calcaneum shell. Several studies have been done to compare the outcome of operative and non-operative management of intraarticular calcaneum fractures. Hilderbrand and Buckley compared forty-four patients treated operatively using a lateral approach with bone grafting with nineteen patients treated conservatively. ${ }^{2}$ The follow up was for 2.9 years. They found significantly better results in the group treated operatively regarding pain, activity level, range of subtalar motion, return to work and radiographic evaluation. Warwick et al reported in a prospective study of twenty-four patients (twelve non-operative and twelve operative procedures) with superior results in patients treated operatively. ${ }^{3}$ Thordarson and Krieger rated their results of twenty-eight patients (seventeen operatives and eleven non-operative). This study reported unequivocally statistically significant superior results in the operative group compared with the non-operative group. ${ }^{4}$ M.Paul et al. (2004) did a review of 70 patients, giving Bohler's angle as a prognostic relevance. They found good results in undisplaced fractures with a normal Bohler's angle which have been treated non-operatively. They found satisfactory result in intra-articular fractures treated operatively with the restoration of the Bohler's angle. ${ }^{5}$ Parmer et al. presented a prospective randomized study involving 56 cases with intraarticular fractures of which 31 were treated operatively by open reduction and $\mathrm{k}$ wire fixation. In the follow-up examination, no statistical difference could be detected between two groups. ${ }^{6}$ A meta-analysis published by Randle et al. (2000) showed no sound evidence which allowed a surgeon to decide upon optimal treatment for intra-articular fracture calcaneum. Jain et al (2007) did their study on 12 patients with intra-articular fracture calcaneum. They developed their post-operative evaluation which is a modification of Myrland Foot score. The study was conducted on calcaneal plating for displaced intra-articular calcaneal fractures. ${ }^{7}$ They concluded that the surgical results were superior in type II and type III fracture and that open reduction and internal fixation methods yield a reasonable 
outcome even in patients with Sanders type IV intra-articular fracture of calcaneus. ${ }^{8}$ Myerson and Quill conducted a study of limited open reduction and internal fixation of displaced intra-articular fractures of the calcaneum. They concluded that this method was significantly shorter with no wound complications. ${ }^{9}$ The limitation of our study is the small sample size and short duration of follow up. More number of similar studies is required to compare the outcomes of our minimally invasive technique using fibula strut graft to that of conventional plating.

\section{CONCLUSION}

This is our innovative minimally invasive technique for tackling comminuted complex depressed fractures of the calcaneum. We achieved satisfactory patient related functional, clinical and radiological outcomes. Fibular stud graft helps in packing the gap, providing stud support as well as cancellous grafting which is giving excellent results of maintaining the heel contour of the proper size, relieving the pain early and providing a virtually good range of movement.

\section{ACKNOWLEDGMENT}

Authors acknowledge the immense help received from the scholars whose articles are cited and included in references to this manuscript. The authors are also grateful to authors /
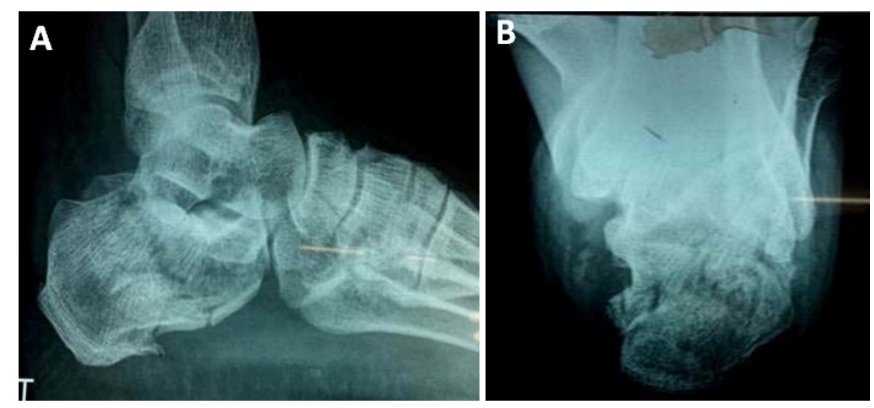

Figure 1: A. Pre-operative lateral view of left calcaneum closed depressed fracture in 26 years female, B. Pre-operative axial view of left calcaneum showing varus deformity.

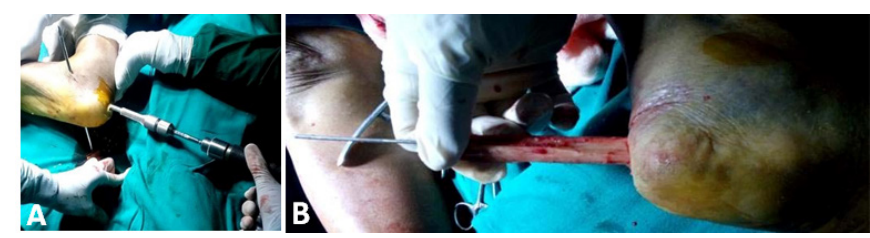

Figure 2: A. Steinman pins insertion in the anterior process of left calcaneum from lateral to medial side for reduction. Triple reamer inserted over the guidewire under image intensifier. B. Pushing of the non-vascularised fibula strut graft over the guide wire. editors / publishers of all those articles, journals, and books from which the literature for this article has been reviewed and discussed.

\section{Conflict of Interest: Nil}

Source of Funding: Nil

\section{REFERENCES}

1. Folk JW, Starr AJ, Early JS. Early wound complications of operative treatment of calcaneus fractures: analysis of 190 fractures. J Orthop Trauma 1999; 13: 369-372.

2. Hilderbrand KA, Buckley RE. Functional outcome measures after displaced intraarticular calcaneum fractures. J Bone Joint Surg 1996; 78:119-123.

3. Warwick CK, Brenner AE. Fractures of the calcaneum. J Bone Joint Surg Br 1953; 35: 33-45.

4. Thordarson DB, Krieger LE. Operative vs nonoperative treatment of intraarticular fractures of the calcaneus: a prospective randomized trial. Foot Ankle Int. 1996; 17: 2-9.

5. Paul M, Peter R, Hoffmeyer P. Fractures of the calcaneum - a review of 70 patients. J Bone Joint Surg [Br] 2004; 86-B: 1142-5.

6. Parmer HV, Triffitt PD, Gregg PJ. Intra-articular fractures of calcaneus treated operatively or conservatively: a prospective study. J Bone Joint Surg 1993; 75B: 932-943.

7. Jain V, Kumar R, Mandal DK. Osteosynthesis for intra-articular calcaneal fractures. Journal of Orthopaedic Surgery 2007; 15(2); 144-8.

8. Sanders R. Intra-articular fracture of the calcaneus: present state of the art. J Orthop Trauma 1992; 6; 252-265.

9. Myerson M, Quill JE: Late complications of fractures of the calcaneus. J Bone Joint Surgery 1993; 15 A: 331-339.


Figure 3: A. Immediate postoperative radiograph of the lateral view left calcaneum, B. Immediate postoperative radiograph of the axial view left calcaneum.
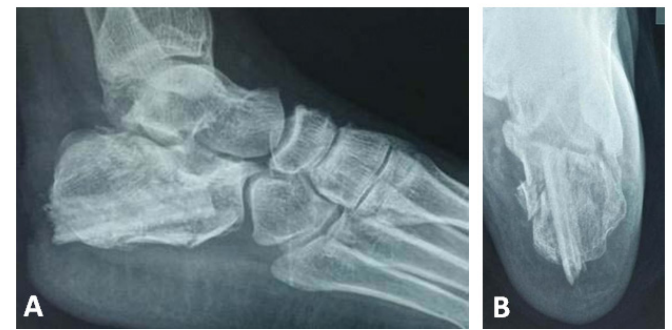

Figure 4: A. 12 weeks follow up lateral view left calcaneum, B. 12 weeks follow up axial view left calcaneum. 


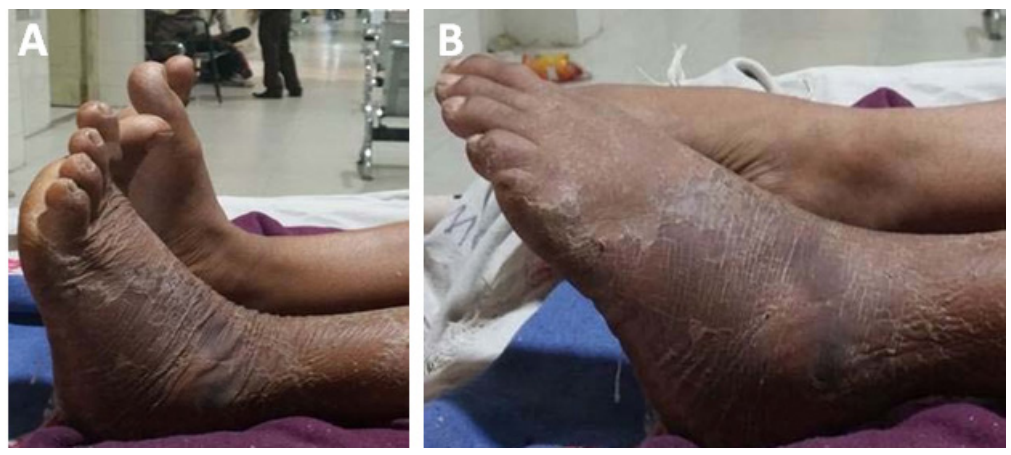

Figure 5: A. Left ankle dorsiflexion at final follow up, B. Left ankle plantarflexion at final follow up.

Table 1: Radiological outcome for patients treated with fibula strut graft for displaced intra-articular calcaneum fractures with varus and sub-talar joint collapse.

\begin{tabular}{llll} 
Radiological Outcome (Mean \pm Standard Deviation) & Pre-operative & $\begin{array}{l}\text { Post-opera- } \\
\text { tive }\end{array}$ & P-value \\
Bohler's Angle $\left(^{\circ}\right)$ & $12 \pm 8$ & $22 \pm 9$ & $<0.05$ \\
Gissane's Angle $\left(^{\circ}\right)$ & $114 \pm 9$ & $121 \pm 7$ & $>0.05$ \\
Calcaneal & $43 \pm 6$ & $45 \pm 5$ & $>0.05$ \\
Height $(\mathrm{mm})$ & & & $<0.05$ \\
Calcaneal Width $(\mathrm{mm})$ & $52 \pm 12$ & $46 \pm 9$ & $<$ \\
\hline
\end{tabular}

